\title{
Dermoscopy of a Tattoo Pseudolymphoma
}

\author{
Mateja Kendel ${ }^{1}$, Ruzica Jurakic Toncic ${ }^{2}$, Mirna Bradamante ${ }^{2}$, Ivana Ilic ${ }^{3}$, \\ Davorin Loncaric ${ }^{2}$, Jaka Rados ${ }^{2}$, Daniela Ledic Drvar ${ }^{2}$
}

\author{
1 Department of Dermatology and Venereology, County Hospital Cakovec, Croatia \\ 2 University Department of Dermatology and Venerology, University Hospital Centre Zagreb, University of Zagreb, School of Medicine, \\ Zagreb, Croatia \\ 3 University Hospital Centre Zagreb, Department of Pathology and Cytology, Zagreb, Croatia
}

Key words: pseudolymphoma, tattoo, dermoscopy

Citation: Kendel M, Jurakic Toncic R, Bradamante M, Ilic I, Loncaric D, Rados J, Ledic Drvar D. Dermoscopy of a tattoo pseudolymphoma. Dermatol Pract Concept. 2019;9(1):17-19. DOI: https://doi.org/10.5826/dpc.0901a04

Published: January 31, 2019

Copyright: $@ 2019$ Kendel et al. This is an open-access article distributed under the terms of the Creative Commons Attribution License, which permits unrestricted use, distribution, and reproduction in any medium, provided the original author and source are credited.

Funding: None.

Competing interests: The authors have no conflicts of interest to disclose.

Authorship: All authors have contributed significantly to this publication.

Corresponding author: Ruzica Jurakic Toncic, MD, University Department of Dermatology and Venerology, University Hospital Centre Zagreb, University of Zagreb, School of Medicine, Salata 4 10000, Zagreb, Croatia. Email: ritoncic@gmail.com

\section{Introduction}

Cutaneous pseudolymphoma is a group of skin disorders with aggravated immunological reaction to known/unknown stimulus [1]. It is a rare complication characterized by inflammatory reaction to tattoo dyes and clinically presents as an erythematous or violaceous pruritic nodule or collection of small nodules in the vicinity of or within the culprit tattoo [1]. Phenotypically, it is a T- or B-cell infiltration and takes a prolonged course to develop, typically several months to years after tattooing [2]. The histopathology of pseudolymphoma often mimics lymphoma. The leading histologic finding is mixed dermal infiltrate of lymphocytes, plasma cells, and eosinophils. Immunohistochemical staining provides a clue showing a polyclonal lymphocyte population (usually found in benign, reactive processes) in contrast to monoclonality found in lymphomas [2]. Although it is a benign skin condition, there have been reports of a malignant transformation due to persistent antigenic stimulation, so careful follow-up is mandatory [2].

\section{Case Presentation}

We report the case of a 35-year-old woman presenting with multiple small, firm, erythematous to violaceous nodules at the periphery of the flower-shaped, multicolored tattoo at her left chest (Figures 1 and 2). The tattoo was made 14 months before skin changes occurred. Based on clinical features, the usual complications of tattoo were suspected, such as keloid, sarcoid granuloma, and foreign body granuloma.

Dermoscopy revealed a nonspecific homogenous violaceous pattern, with nonsharp border at the periphery. Few lesions showed follicular accentuation (follicular whiteyellow halo). Lesions were negative for yellow-orange background typical of the diagnosis of granulomatous skin disorders, allowing us to exclude them from differential diagnosis. Lesions had a violet pink color, but without criteria for vascular lesions (Figures 3-6).

Differential diagnosis also included lymphoma and cutaneous metastases; therefore, an excision of one nodule was done. Histology showed thin epidermis with orthokeratosis, 


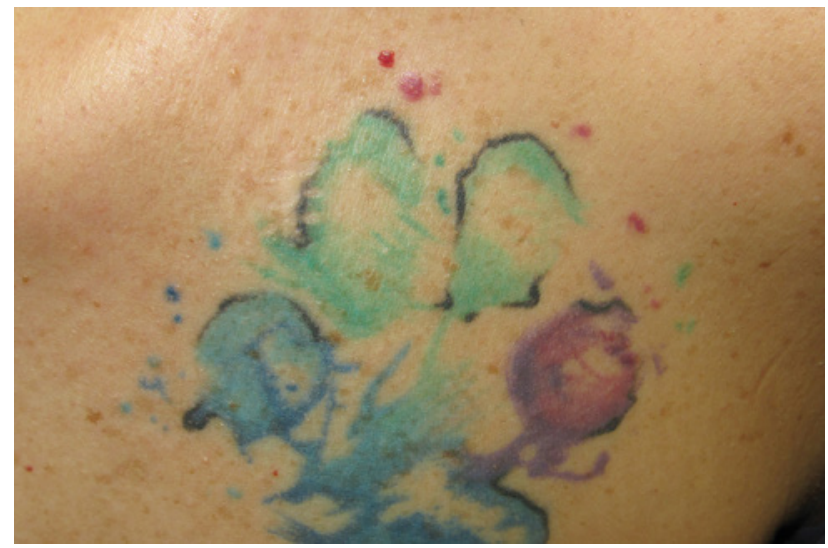

Figure 1. Clinical presentation of a tattoo pseudolymphoma (image taken with Canon Power Shot SX520HS). [Copyright: (02019 Kendel et al.]

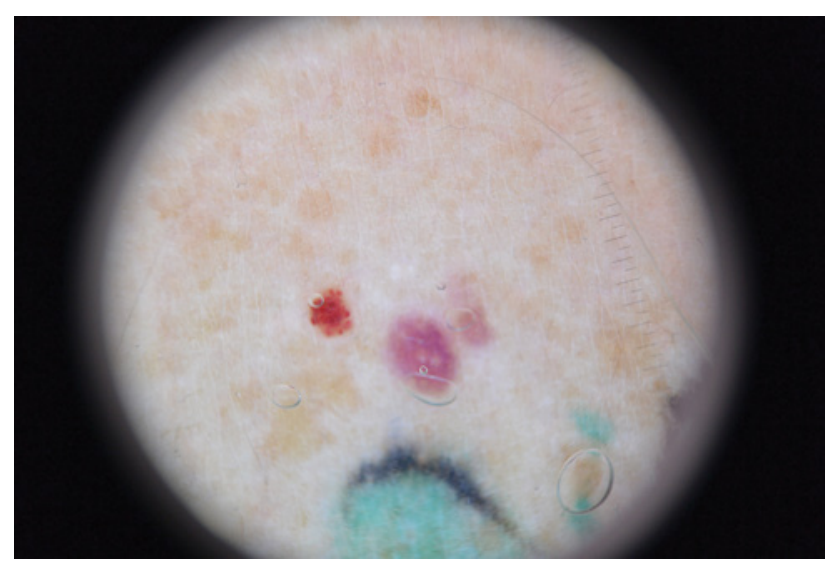

Figure 3. Dermoscopic image of a tattoo pseudolymphoma (Dermlite Photo Nikon AW1). [Copyright: (2019 Kendel et al.]

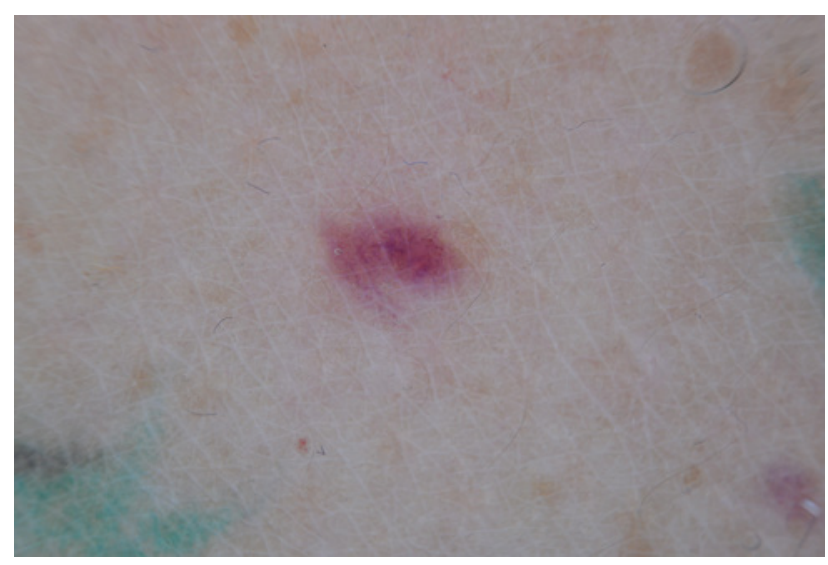

Figure 5. Dermoscopic image of a tattoo pseudolymphoma (Dermlite Photo Nikon AW1). [Copyright: (C2019 Kendel et al.]

overlying a dense dermal infiltrate of lymphocytes of small and medium size, without nuclear atypia. In addition to lymphocytes, the mixed infiltrate also contained histiocytes and exogenous red pigment. Immunohistochemistry confirmed the infiltrate mostly of CD3 $+\mathrm{T}$ lymphocytes with

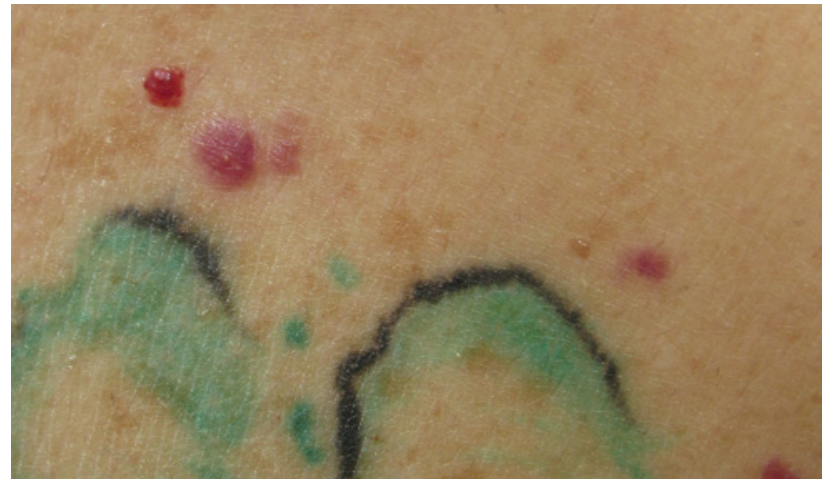

Figure 2. Clinical presentation of a tattoo pseudolymphoma (image taken with Canon Power Shot SX520HS). [Copyright: (92019 Kendel et al.]

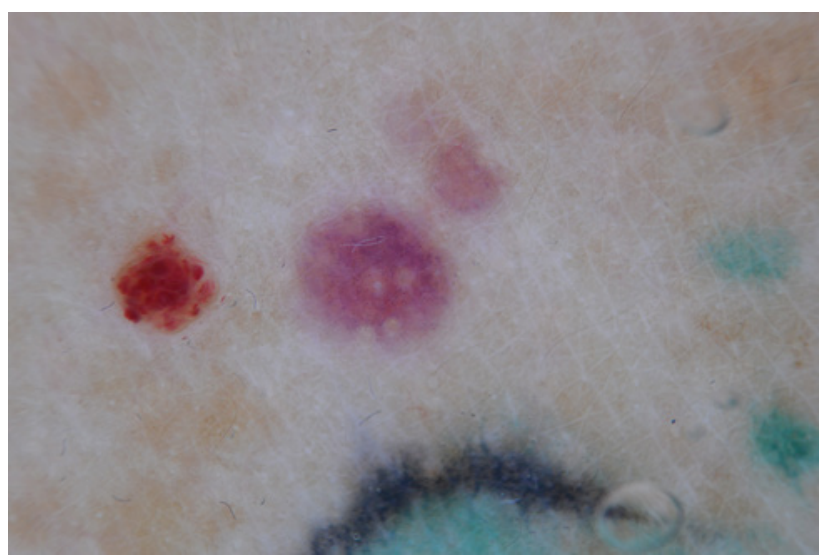

Figure 4. Dermoscopic image of a tattoo pseudolymphoma (Dermlite Photo Nikon AW1). [Copyright: (02019 Kendel et al.]

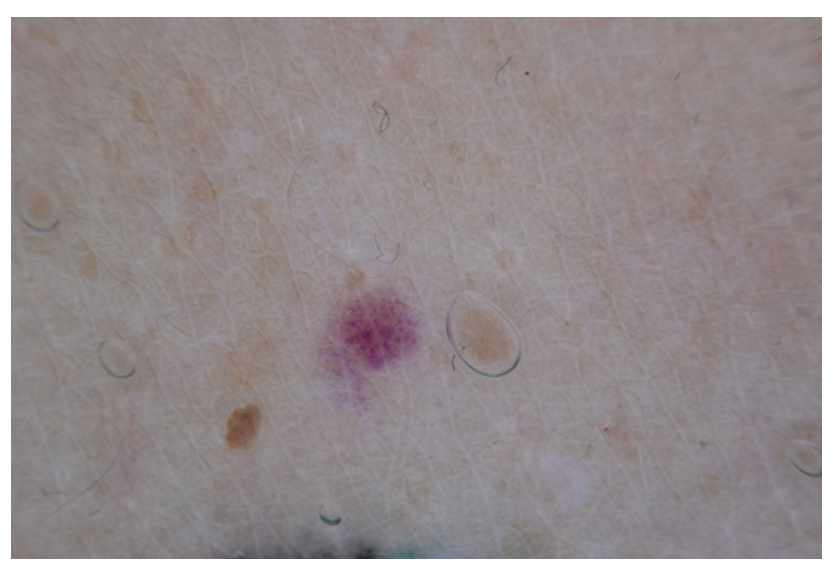

Figure 6. Dermoscopic image of a tattoo pseudolymphoma (Dermlite Photo Nikon AW1). [Copyright: @2019 Kendel et al.]

few scattered CD20+ B cells. The plasma cell population showed a polytypic pattern of immunoglobulin light-chains. The histological architectural pattern, lack of atypia, and immunohistochemistry suggested the diagnosis of T-cell pseudolymphoma (Figures 7 and 8). 


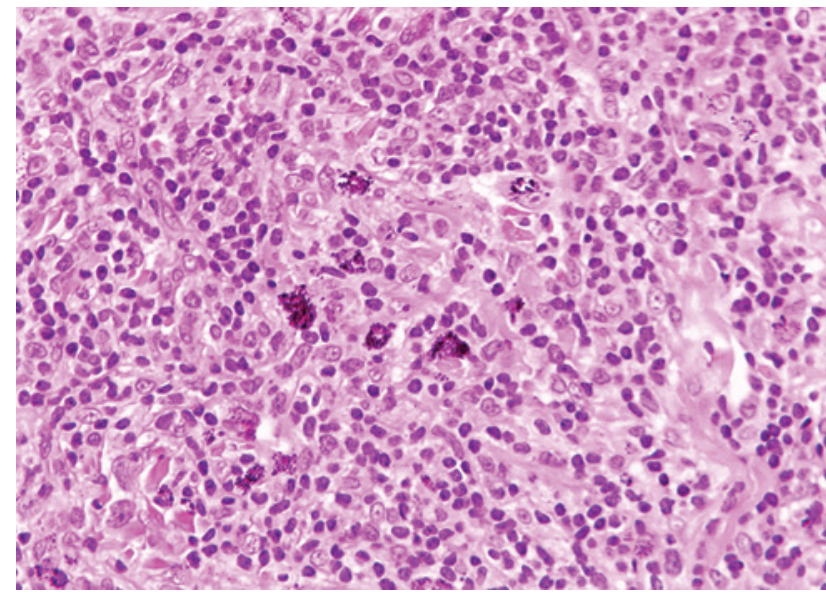

Figure 7. Histological findings in a tattoo pseudolymphoma. [Copyright: @2019 Kendel et al.]

\section{Conclusions}

Tattoo pseudolymphoma is considered as a rare benign disease and can be treated with topical/intralesional corticosteroids, surgical excision, or laser treatment [2]. Because the clavicular region is prone to the development of keloids, we chose close follow-up. Keeping in mind the popularity of tattoos, we can expect more complications related to them, one of which is certainly pseudolymphoma development.

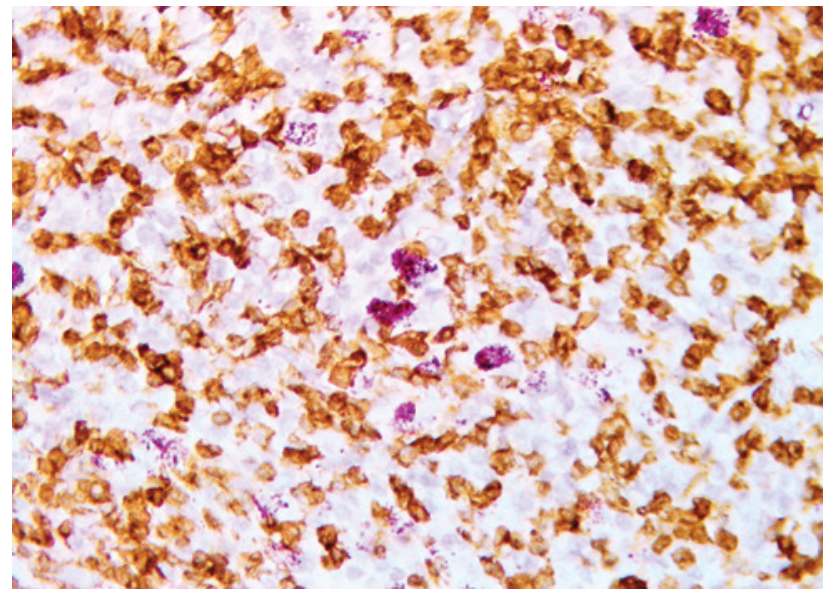

Figure 8. Immunohistochemical analysis of a tattoo pseudolymphoma. [Copyright: (02019 Kendel et al.]

\section{References}

1. Kluger N, Vermeulen C, Moguelet P, et al. Cutaneous lymphoid hyperplasia (pseudolymphoma) in tattoos: a case series of seven patients. J Eur Acad Dermatol Venereol. 2010;24(2):208-213.

2. Bergman R. Pseudolymphoma and cutaneous lymphoma: facts and controversies. Clin Dermatol. 2010;28(5):568-574. 